\title{
Regulation of myeloproliferation and M2 macrophage programming in mice by Lyn/Hck, SHIP, and Stat5
}

\author{
Wenbin Xiao, ${ }^{1}$ Hong Hong, ${ }^{1}$ Yuko Kawakami, ${ }^{1}$ Clifford A. Lowell, ${ }^{2}$ and Toshiaki Kawakami ${ }^{1}$ \\ 1Division of Cell Biology, La Jolla Institute for Allergy and Immunology, La Jolla, California, USA. \\ 2Department of Laboratory Medicine, UCSF, San Francisco, California, USA.
}

\begin{abstract}
The proliferation and differentiation of hematopoietic stem cells (HSCs) is finely regulated by extrinsic and intrinsic factors via various signaling pathways. Here we have shown that, similar to mice deficient in the lipid phosphatase SHIP, loss of 2 Src family kinases, Lyn and Hck, profoundly affects HSC differentiation, producing hematopoietic progenitors with increased proliferation, reduced apoptosis, growth factor-independent survival, and skewed differentiation toward M2 macrophages. This phenotype culminates in a Stat5-dependent myeloproliferative disease that is accompanied by M2 macrophage infiltration of the lung. Expression of a membrane-bound form of SHIP in HSCs lacking both Lyn and Hck restored normal hematopoiesis and prevented myeloproliferation. In vitro and in vivo studies suggested the involvement of autocrine and/or paracrine production of IL-3 and GM-CSF in the increased proliferation and myeloid differentiation of HSCs. Thus, this study has defined a myeloproliferative transformation-sensitive signaling pathway, composed of Lyn/Hck, SHIP, autocrine/paracrine cytokines, and Stat5, that regulates HSC differentiation and M2 macrophage programming.
\end{abstract}

\section{Introduction}

The Src family kinases (SFKs) mediate signals from a multitude of cell surface receptors to regulate a variety of cellular functions including proliferation, differentiation, and migration (1). Several SFK members are expressed in hematopoietic cells and linked to signaling by a number of cytokine receptors and receptor tyrosine kinases. Thus, SFKs play important roles in the development and function of hematopoietic cells: for example, $l y n^{-/-}$mice suffer from a deficit of mature B cells $(2,3)$. However, aged $\mathrm{lyn}^{-/-}$mice display autoimmune disease and perturbations in myelopoiesis that ultimately lead to splenomegaly and macrophage tumors (4). Impaired hematopoiesis in lyn $n^{-/-}$mice is explained by the essential role of Lyn in establishing immunoreceptor tyrosine-based inhibitory motif-dependent (ITIM-dependent) signaling. ITIMcontaining inhibitory receptors phosphorylated by Lyn recruit protein tyrosine phosphatases, such as $\mathrm{SH} 2$-domain containing phosphatase-1 (SHP-1) and SHP-2 as well as SH2-domain containing $5^{\prime}$-inositol phosphatase (SHIP-1), to the plasma membrane (5). Perturbations in these signaling pathways result in an increased number of myeloid progenitors (6) and impaired B-lymphopoiesis in lyn ${ }^{-1-}$ mice. The SFKs Hck and Fgr also serve an inhibitory function in hematopoiesis and mature leukocyte function. Deficiency of Hck alone results in increased G-CSF induced proliferation of granulocytic precursors (6), while loss of both kinases results in

Nonstandard abbreviations used: BAL, bronchoalveolar lavage; $\mathrm{CML}$, chronic myelogenous leukemia; CMP, common myeloid progenitor; dko, double-knockout; DN, dominant-negative; G-CFU, granulocyte-CFU; GMP, granulocyte-macrophage progenitor; KSL, c-Kit ${ }^{+}$Sca-1 $1^{+}$lineage ${ }^{-}$LT-HSC, long-term HSC; Mix-CFU, granulocyte/erythrocyte/monocyte/megakaryocyte-CFU; MPD, myeloproliferative disease; SFK, Src family kinase; SHIP, SH2-domain containing 5'-inositol phosphatase; SHP-1, SH2-domain containing phosphatase-1; ST-HSC, short-term HSC.

Conflict of interest: The authors have declared that no conflict of interest exists. Citation for this article: J. Clin. Invest. 118:924-934 (2008). doi:10.1172/JCI34013. exaggerated chemokine-induced activation in neutrophils and dendritic cells (7). Despite these observations, roles of SFKs in HSC homeostasis are poorly defined.

SHIP is expressed primarily in hematopoietic cells including HSCs $(8,9)$. This enzyme hydrolyzes the 5 ' phosphate on phosphatidylinositol 3,4,5-trisphosphate and inositol 1,3,4,5-tetrakisphosphate, thus preventing membrane recruitment and activation of pleckstrin homology domain-containing kinases that serve as effectors of phosphatidylinositol-3'-kinase (PI3K) signaling. SHIP associates with various adaptor/scaffold proteins or inhibitory receptors following activation of hematopoietic cells. SHIP-deficient mice exhibit chronic hyperplasia of myeloid cells resulting in splenomegaly, lymphadenopathy, and myeloid infiltration of lung and other organs $(10,11)$. Neutrophils and bone marrow-derived (BM-derived) mast cells from $\mathrm{SHIP}^{-/-}$mice are resistant to apoptosis. Consistent with these observations, HSC proliferation and numbers are increased in SHIP $\mathrm{P}^{-/-}$mice (12). Thus, SHIP limits the survival, activation, proliferation, and differentiation of hematopoietic cells.

Here we show that mice deficient in Lyn and Hck develop myeloproliferative disease (MPD) accompanied by severe lung inflammation with extensive macrophage infiltration, and that both the MPD and the lung inflammation originate from long-term HSCs (LT-HSCs). Our study has also revealed a signaling pathway for Lyn/ Hck/SHIP-mediated inhibition of cytokines production that, via the Stat5 signaling, tightly controls the proliferation, apoptosis, and differentiation of HSCs and myeloid progenitors. Perturbations of this pathway lead to myeloproliferative transformation of LT-HSCs.

\section{Results}

Lyn/Hck-deficient mice suffer from severe lung inflammation. During

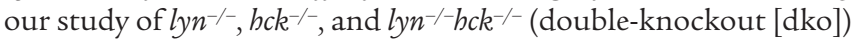
mice, we noticed that dko mice died prematurely. Dko mice were 
A
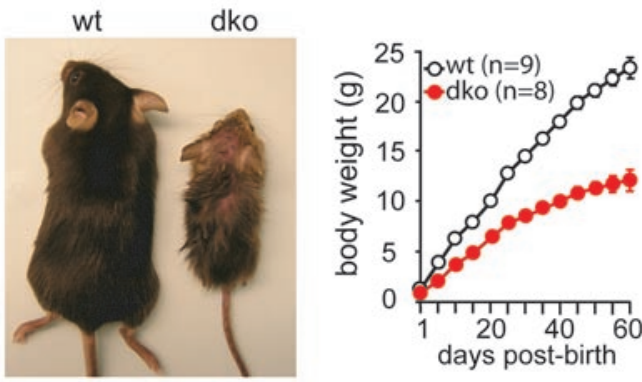

C
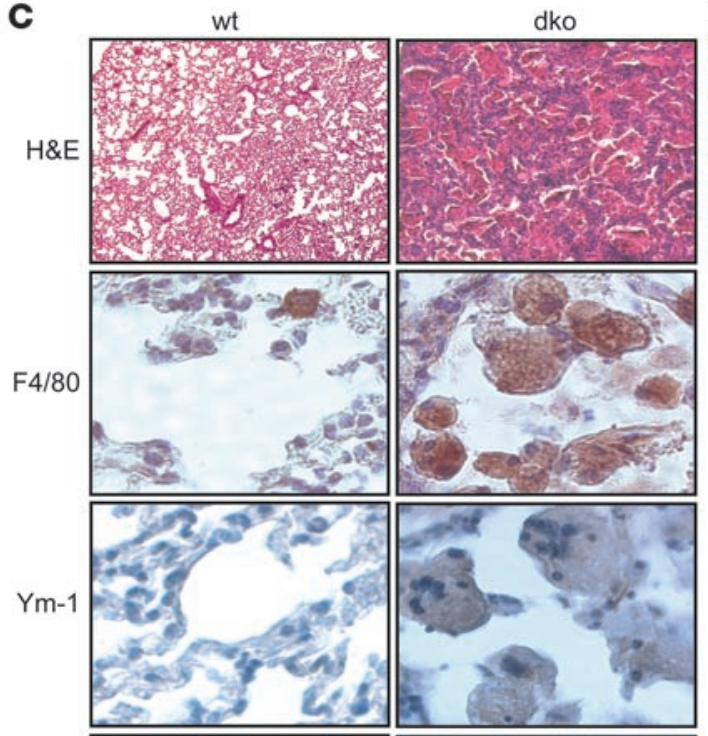

Arg I

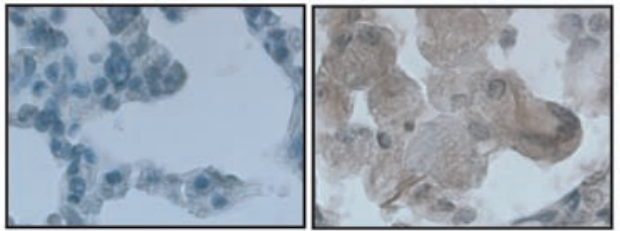

B

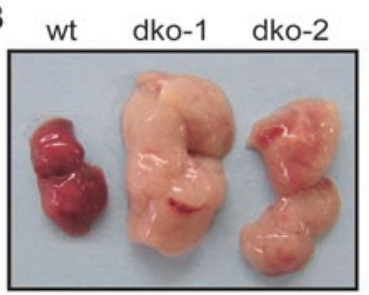

D dko $\times 400$

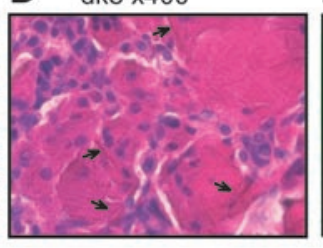

$\mathbf{F}$

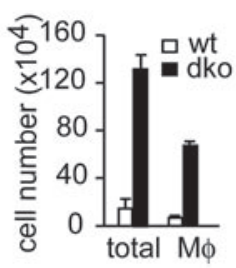

G

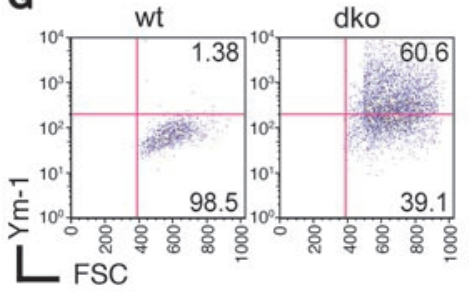

Figure 1

Severe lung inflammation characterized by extensive infiltration with M2type macrophages in Lyn/Hck-deficient mice. (A) Growth of dko mice was slower than WT mice. The mice shown are 8 weeks in age. Data represent mean \pm SD throughout the figures. (B) Gross appearance showed that lungs are consolidated in dko mice. (C and D) Microscopic examination showed abundant M2 macrophages in alveoli of dko lungs. Original magnification, $\times 100(\mathrm{H} \& \mathrm{E})$; others, $\times 400$ (C). Arrows indicate crystals. (E) A crystal recovered from BAL from dko mice and illuminated by green light of $488 \mathrm{~nm}$ (original magnification, $\times 400)$. (F) Increased numbers of macrophages $(\mathrm{M} \phi)$ were recovered in BAL fluids of dko mice. (G) Ym-1-positive cells were increased in BAL macrophages from dko mice. born at normal Mendelian frequency (Supplemental Figure 1; supplemental material available online with this article; doi:10.1172/ JCI34013DS1); however, within 2 weeks they became obviously sick, growth retarded, and most died within 2 months (Figure 1A). By contrast, $l \mathrm{yn}^{-/-}$and $h c k^{-/-}$mice remained healthy for the first 2 months of age (data not shown). Gross examination revealed that the lungs in dko mice were bilaterally consolidated (Figure 1B). Histologically, the lungs were fibrotic and profoundly infiltrated with inflammatory cells, the majority of which were $\mathrm{F} 4 / 80^{+}$macrophages (Figure 1, C and D, and Supplemental Figure 2). Compared with WT mice, total cell numbers in bronchoalveolar lavages (BALs) were increased by 10-fold in dko mice (Figure 1F). Fifty-five percent of the cells were macrophages, and most other cells (32\%) were $\mathrm{CD} 11 \mathrm{~b}^{+} \mathrm{Gr}-1^{-} \mathrm{F} 4 / 80^{-}$monocytes (data not shown). These macrophages expressed Ym-1 and arginase I (Figure 1, C and G), suggesting an M2-skewed phenotype $(13,14)$. Similar to those in $S_{H I P^{-/-}}$mice $(10,11,15)$, eosinophilic hexagonal crystals were abundantly found in BAL fluids recovered from dko, but not WT, mice (Figure 1E). Given the extensive nature of lung inflammation, this could be a cause of premature deaths in dko mice.

Dko mice develop myeloproliferative disease. In addition to the macrophage/monocyte pulmonary inflammation, dko mice displayed splenomegaly (Supplemental Figure 3, A and B). A dramatic increase of granulocytes in BM, spleen, and peripheral blood from dko mice was revealed by flow cytometric analysis (Figure 2, A and B) and differential blood cell counting (Supplemental Figure $3 \mathrm{D})$. Leukocyte numbers in peripheral blood were increased by 3 -fold $(21,750 \pm 3,780 / \mu \mathrm{l}$ in dko mice versus 6,850 $\pm 540 / \mu \mathrm{l}$ in WT mice; $P<0.001 ; n=5)$ in 2 -month-old mice. Most of the increased leukocytes were mature granulocytes (Figure 2C). Monocytes $\left(\mathrm{CD} 11 \mathrm{~b}^{+} \mathrm{Gr}-1^{-}\right)$in peripheral blood were also increased (Figure 2A and Supplemental Figure 3D). In addition to pulmonary infiltration, dko mice also showed evidence of hepatic infiltration by myeloid cells (Supplemental Figure 3C). These data collectively indicate that all dko mice analyzed $(n=12)$ exhibit a set of clinical signs within 2 months of age that fulfill the criteria for the MPD (16). No abnormalities in myelopoiesis were observed with either $l y n^{-/-}$or $b c k^{-/-}$mice for the first 2 months after birth, whereas old (>6 months of age) $l y n^{-/}$, but not $h c k^{-/-}$, mice showed a mild MPD phenotype (ref. 17 and data not shown).

Dko mice have increased HSCs and myeloid progenitors. Given their MPD phenotype, we investigated the hematopoietic process in dko mice in more detail. Flow cytometric analysis revealed that the absolute number of $\mathrm{c}-\mathrm{Kit}^{+} \mathrm{Sca}-1^{+}$lineage- $(\mathrm{KSL})$ cells, which are 
A
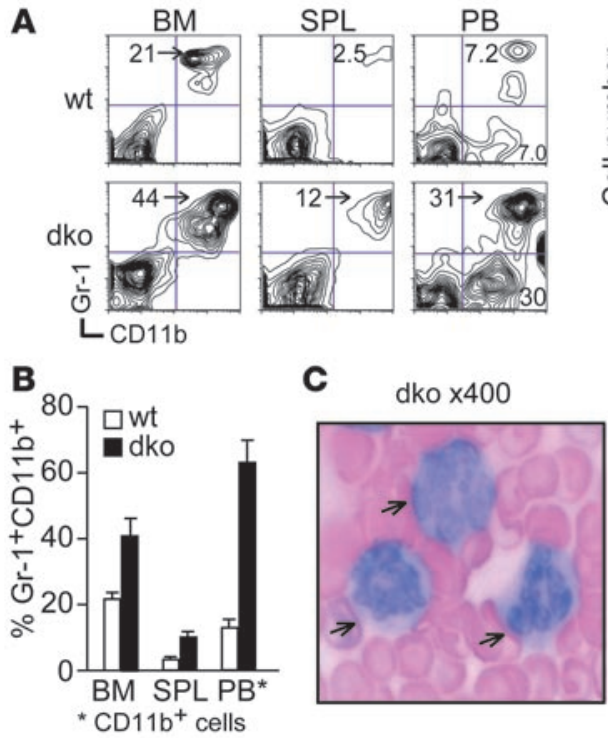

D
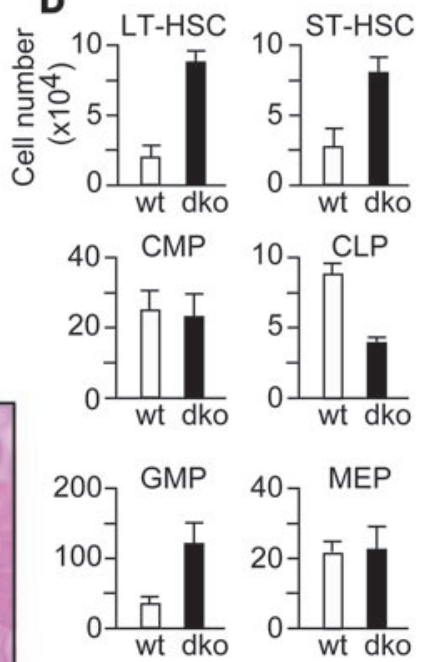

Figure 2

The MPD phenotype in Lyn/Hck-deficient mice. (A and B) Increased myeloid cells (Gr-1+CD11b+ or CD11b+) were found in BM, spleen (SPL), and peripheral blood (PB) from dko mice, as quantified by flow cytometry. (C) Shown is a Wright-Giemsa-stained blood smear of dko mouse (original magnification, $\times 400$ ). Arrows indicate neutrophils. (D) Accumulated flow cytometric data of BM cells are shown $(n=12)$. Numbers of specific cell types were calculated by multiplying their percentages (obtained by flow cytometry; see Supplemental Figure 4) by total cell numbers. Dko mice have increased numbers of HSCs and myeloid progenitors but decreased numbers of lymphoid progenitors (see Supplemental Figures 4 and 5). MEP, megakaryocyte-erythroid progenitor. enriched for HSCs, in BM from dko mice is increased by 4.1-fold compared with WT mice (Figure 2D and Supplemental Figure 4). Further, dko mice had 4.2-fold more KSL CD34- cells (enriched for long-term HSCs [LT-HSCs]; ref. 18) and 3.2-fold more KSL CD34+ cells (enriched for short-term HSCs [ST-HSCs]). Granulocyte-macrophage progenitors (GMPs) were also increased by 3 -fold. However, both common myeloid progenitors (CMPs) and megakaryocyte-erythroid progenitors (MEPs) were comparable in WT and dko mice. By contrast, the number of common lymphoid progenitors was decreased by 2-fold (Figure 2D), consistent with reduced $\mathrm{T}$ and $\mathrm{B}$ cells in dko mice (Supplemental Figure 5). Compared with WT cells, BM cells from dko mice formed increased numbers of myeloid colonies (i.e., granulocyte-CFU [G-CFU], M-CFU, GM-CFU, and granulocyte/erythrocyte/ monocyte/megakaryocyte-CFU [MixCFU]) in methylcellulose medium containing IL-3, IL-6, stem cell factor (SCF), and erythropoietin, but reduced numbers of pre-B cell colonies in medium containing IL-7 (Supplemental Figure 4, B and C). Overall, lyn/hck dko mice had increased HSCs and GMPs, similar to other mouse MPD models (19-21), suggesting that the MPDs in these mice may be caused by similarly perturbed hematopoiesis (Supplemental Figure 4D).

$M P D$ and lung disease originate from LT-HSCs in dko mice. A cardinal feature of the mouse MPD is the transplantability of disease (19). Therefore, we performed a series of transplantation experiments (Table 1). First, BM cells from WT or dko mice (Ly5.2) were i.v. injected into lethally

Table 1
MPD and lung inflammation are transplantable with LT-HSCs of Lyn/Hck-deficient mice

\begin{tabular}{|c|c|c|c|c|}
\hline \multirow[t]{2}{*}{ Donor cells } & \multicolumn{2}{|c|}{ Engraftment ${ }^{A}$} & \multirow[t]{2}{*}{$\%$ Gr-1+CD11b+ } & \multirow{2}{*}{$\begin{array}{l}\text { Myeloid hype } \\
\text { lung infiltra } \\
\text { per recipient }\end{array}$} \\
\hline & 3 wk & 9 wk & & \\
\hline \multicolumn{5}{|l|}{ BM cells } \\
\hline WT & nd & $>99$ & $18.5 \pm 5.2$ & $0 / 8$ \\
\hline dko & nd & $>99$ & $71.0 \pm 11.0$ & $12 / 12$ \\
\hline \multicolumn{5}{|l|}{ LT-HSC } \\
\hline WT & $54.0 \pm 11$ & $58.0 \pm 15$ & $22.0 \pm 4.3$ & $0 / 6$ \\
\hline dko & $46.7 \pm 4.3$ & $49.1 \pm 5.6$ & $78.4 \pm 3.3$ & $8 / 8$ \\
\hline dko+vec & $33.0 \pm 13$ & $38.0 \pm 14$ & $68.0 \pm 8.7^{B}$ & $6 / 6$ \\
\hline $\mathrm{dko+DN5}$ & $10.0 \pm 0.8$ & $9.0 \pm 1.8$ & $21.4 \pm 6.2^{B}$ & $0 / 8$ \\
\hline \multicolumn{5}{|l|}{ ST-HSC } \\
\hline WT & $51.2 \pm 3.2$ & $11.4 \pm 4.5$ & $5.9 \pm 2.6$ & $0 / 4$ \\
\hline dko & $41.0 \pm 9.4$ & $9.0 \pm 5.1$ & $4.1 \pm 1.5$ & $0 / 4$ \\
\hline \multicolumn{5}{|l|}{ CMP } \\
\hline WT & $3.2 \pm 1.6$ & 0 & 0 & $0 / 4$ \\
\hline dko & $3.4 \pm 0.6$ & 0 & 0 & $0 / 4$ \\
\hline \multicolumn{5}{|l|}{ GMP } \\
\hline WT & $8.3 \pm 3.5$ & 0 & 0 & $0 / 4$ \\
\hline dko & $0.8 \pm 0.4$ & 0 & 0 & $0 / 4$ \\
\hline
\end{tabular}

The MPD and lung phenotypes were transplantable with BM or KSL CD34- cells (LT-HSCs). These phenotypes were prevented by expression of DN Stat5 in LT-HSCs. BM cells $\left(2 \times 10^{6}\right)$, LT-HSCs $\left(1 \times 10^{4}\right)$, ST-HSCs $\left(2 \times 10^{4}\right)$, CMPs $\left(2 \times 10^{4}\right)$, GMPs $\left(2 \times 10^{4}\right)$, or transduced LT-HSCs were i.v. transferred into lethally irradiated Ly5.1 mice. LT-HSCs and other fractionated cells were cotransferred with $2 \times 10^{5}$ Sca-1- BM cells of Ly5.1 mice. Peripheral blood cells were analyzed 3 and 9 weeks after transplantation. The fourth column shows percent $\mathrm{Gr}-1+\mathrm{CD} 11 \mathrm{~b}{ }^{+}$cells among the donorderived cells at the ninth week as determined by staining for Ly5.2. Dying mice were sacrificed, and the MPD phenotype was confirmed by flow cytometry of BM and spleen cells. Lung inflammation was detected by histological analysis. All the mice that developed MPD also developed lung infiltration with macrophage infiltrates. Transplantation results were combined from 2 independent experiments. A\%CD45.2+ cells. ${ }^{\mathrm{B}} \mathrm{CD} 11 \mathrm{~b}+$ cells. 

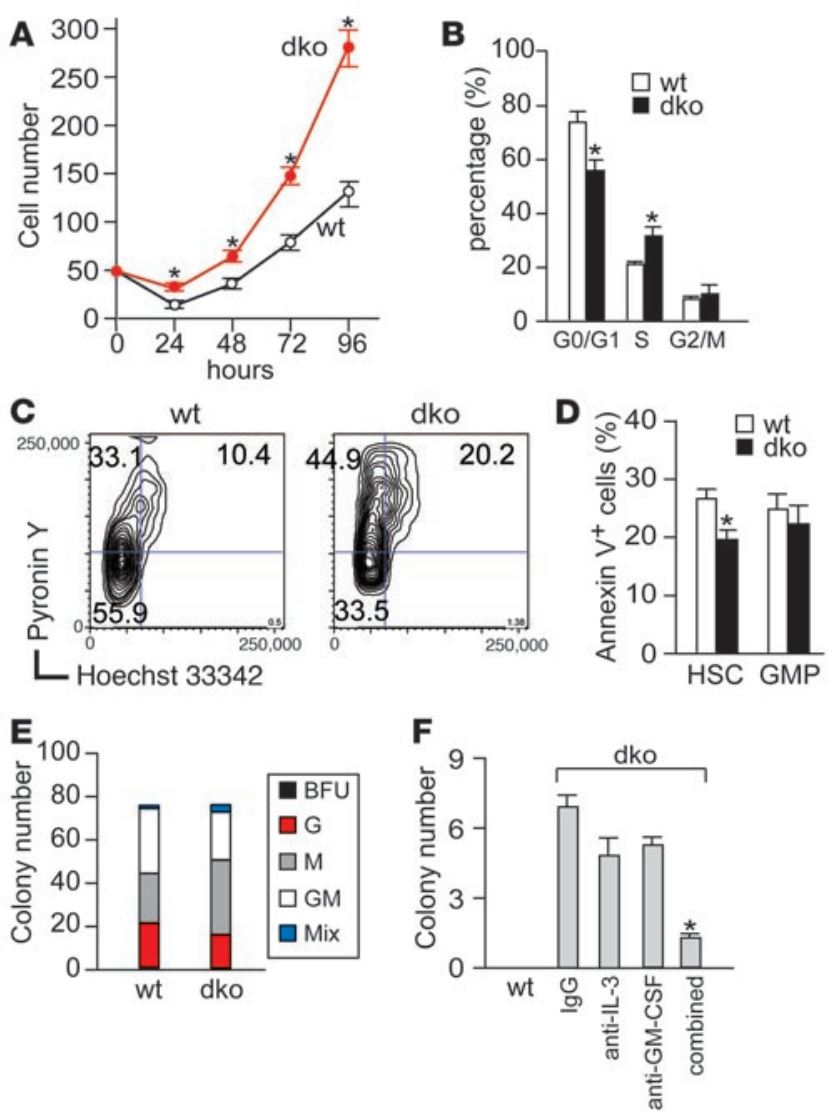

that both the MPD and lung disease are BM cell-autonomous. By contrast, none of the mice that had received WT BM cells developed MPD or lung disease. Next, purified subpopulations of BM cells from dko or WT mice were injected into lethally irradiated C57BL/6-Ly5.1 mice to identify the precise BM compartment that is responsible for the development of MPD and lung disease in $\mathrm{dko}$ mice. All the mice that had received dko, but not WT, LT-HSCs developed both MPD and lung disease within 9 weeks (Table 1). By contrast, the mice that had received ST-HSCs, CMPs, or GMPs derived from dko or WT mice failed to develop either disease, although there were no significant differences in engraftment of LT-HSC, ST-HSC, or CMP between WT and dko recipients 3 weeks after transplant (Table 1). The engraftment of GMP derived from dko mice was even lower than that of WT GMP. These results strongly suggest that the MPD and lung inflammation in dko mice originates from LT-HSCs, although we cannot rule out the possibility that other hematopoietic populations of dko mice might have the ability to cause the diseases. For example, transplantation with larger numbers of ST-HSCs or myeloid progenitors of dko mice might be able to cause the diseases in recipient mice.

HSCs from dko mice proliferate and differentiate into macrophages more vigorously and apoptose less abundantly than WT HSCs. The increase of HSCs in dko mice could be due to an increased proliferation, decreased apoptosis, altered differentiation, or a combination of these factors. In fact, both KSL cells and KSL CD34- cells from $\mathrm{dko}$ mice grew faster under various in vitro culture conditions than WT cells (Figure 3A and data not shown). Consistent with this, cell cycle analysis showed increased proportions of dko KSL cells in the $G_{1}$ and $S$ phases and reduced proportions in the $G_{0}$

\section{Figure 3}

HSCs from dko mice exhibit increased proliferation, decreased apoptosis, and skewed differentiation into macrophages. (A) KSL CD34- cells derived from WT and dko mice were sorted into a 96-well plate containing SCF, Flt3 ligand, and IL-11, and cultured for 4 days. ${ }^{*} P<0.05$ versus WT values by Student's $t$ test. (B and $\mathbf{C}$ ) Cell cycle analysis of $\mathrm{KSL}$ cells was performed by flow cytometry of cells stained with propidium iodide (B) or with Hoechst 33342 and Pyronin $Y(\mathbf{C})$. Lower left, upper left, and upper right quadrants in panel $\mathbf{C}$ contain cells in $\mathbf{G}_{0}, \mathrm{G}_{1}$, and $S / G_{2} / M$ phases, respectively. $G_{0}$, WT $55.1 \% \pm 0.8 \%$ versus dko $34.2 \% \pm 0.7 \%, P=0.002 ; \mathrm{G}_{1}, 31.8 \% \pm 1.4 \%$ versus $45.0 \% \pm 0.1 \%$, $P=0.01 ; \mathrm{S} / \mathrm{G}_{2} / \mathrm{M}, 10.1 \% \pm 0.3 \%$ versus $20.9 \% \pm 0.7 \%, P=0.004$. (D) Flow cytometric analysis indicated reduced annexin $\mathrm{V}^{+}$apoptotic cells in KSL (HSC), but not in GMPs, derived from BM of dko mice. (E) Colony-forming assays showed skewed differentiation of KSL cells (100 cells seeded) of dko mice into macrophages in the presence of IL-3, IL-6, SCF, and erythropoietin. (F) Dko KSL cells (50 cells/well seeded), unlike WT cells, formed colonies in methylcellulose without growth factors, which was inhibited by anti-IL-3 and anti-GM-CSF antibodies. BFU, BFU-E; G, G-CFU; M, M-CFU; GM, GM-CFU; Mix, Mix-CFU.

phase (Figure 3, B and C). Moreover, fewer numbers of dko KSL cells stained with annexin $\mathrm{V}$, indicating that dko HSCs are more resistant to apoptosis (Figure 3D). Finally, colony-forming assays showed that dko KSL cells generated more monocyte/macrophage (M-CFU) colonies than WT cells, indicating that dko HSCs have an increased predisposition to differentiate into monocytes and macrophages (Figure 3E). Moreover, a small population $(-14 \%)$ of KSL cells formed GM-CFU colonies spontaneously in the absence of growth factors (Figure 3F), indicating that these progenitors contained some transformed cells. Taken together, the proliferation, survival, differentiation, and myeloproliferative transformation of HSCs are tightly regulated by Lyn and Hck.

Increased Stat5 activation is essential for increased proliferation and granulocyte/macrophage differentiation of HSCs in dko mice. To dissect the molecular mechanism for the increased proliferation, survival, and myeloid differentiation of dko HSCs, we examined several signaling pathways, including MAPKs, Akt, and Jak-Stat, which work as signal mediators for proliferation and survival of $\operatorname{HSCs}(22,23)$. Although there is no significant difference in phosphorylation of ERK1/2, p38, and Stat3 (Supplemental Figure 7), phosphorylation of Akt, a serine/threonine kinase critical for cell survival (reviewed in ref. 24), was modestly increased in dko KSL cells compared with WT cells, consistent with the modestly increased survival of the former cells. Importantly, phosphorylation levels of Stat5 at Tyr694 were dramatically increased in unstimulated and cytokinestimulated dko KSL cells (Figure 4A). Flow cytometry and imaging analysis demonstrated that KSL cells with nuclear localization of phosphorylated Stat5 (type II cells in Figure 4B) were more frequently found in dko mice. Similar results with Stat 5 phosphorylation were also obtained with BAL macrophages from dko mice (Figure 4, C-E). mRNA and protein levels of Stat5 (both Stat5A and Stat5B) were comparable in dko and WT KSL cells (data not shown). Consistent with Stat5 activation, mRNA levels of the well-known Stat 5 targets, e.g., cytokine-inducible SH2-containing protein (CIS) (25), pim-1 (26) (a serine/threonine kinase that can promote cytokine-independent survival; ref. 27), and oncostatin M (28), were all increased in dko cells (Figure 4F, upper panel). These results indicate that Stat 5 is constitutively activated in dko HSCs.

To directly test the functional relevance of the increased Stat5 activity, we introduced dominant-negative (DN) Stat5 into WT 
A

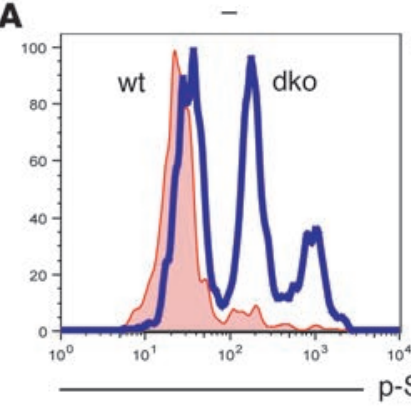

C

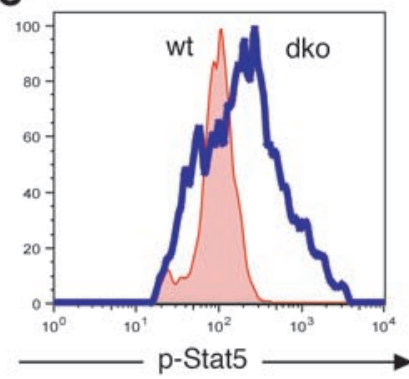

$\mathbf{F}$
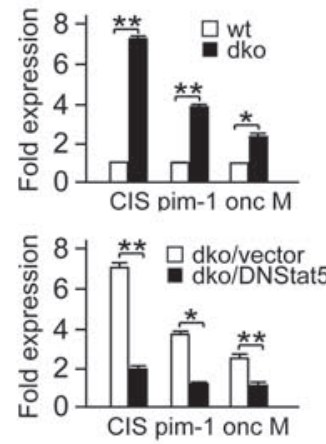

$+$

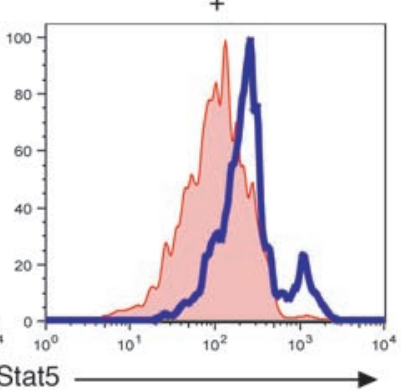

B

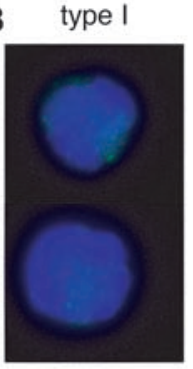

type II

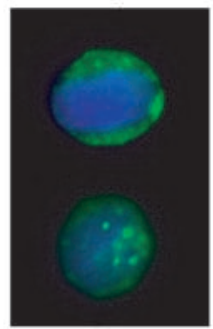

$1+11$

wt: $90+10$

dko: $29+71$
D

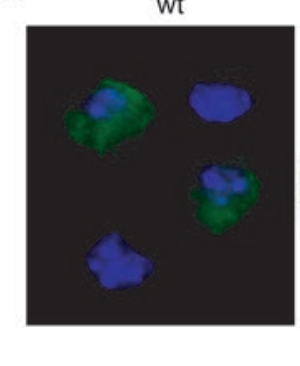

E

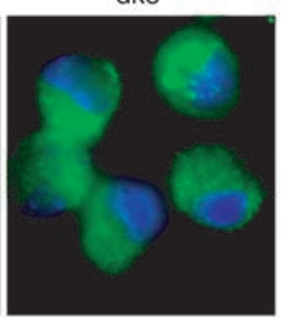

wt

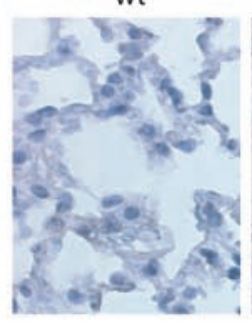

dko

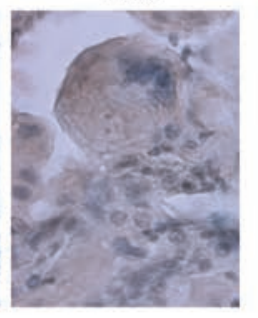

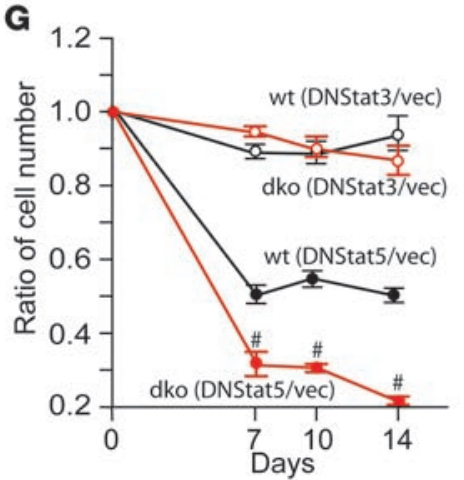

H

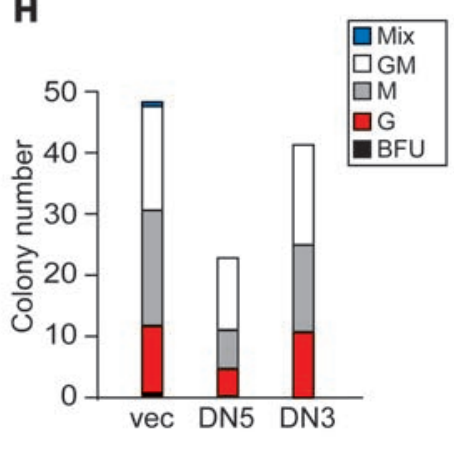

Figure 4

Stat5 activation is required for the increased proliferation and macrophage differentiation of KSL cells from Lyn/Hck-deficient mice. (A and B) Stat5 in dko KSL cells was constitutively activated (-), and its activity was further increased upon stimulation with a cytokine cocktail of IL-3, SCF, and Flt3 ligand (+), as shown by flow cytometric analysis (A) and confocal microscopy (B) using anti-p-Stat5 (Tyr694) antibody. Nuclei were stained with DAPI (blue). Original magnification, $\times 400$. One hundred cells were scored for either the absence (type I) or presence (type II) of anti-p-Stat5 staining and those numbers are shown to the right of the panel. (C-E) Stat5 in dko lung macrophages was constitutively phosphorylated, as shown by flow cytometric analysis (C), confocal microscopy (D), and immunohistochemistry (E). Original magnification, $\times 400$ (D and E). (F) Real-time PCR was performed for mRNA expression of Stat5 target genes, cytokine-inducible SH2-containing protein (CIS), pim-1, and oncostatin $M$ (onc M). Expression was normalized against L32 RNA and set at 1 in WT cells. ${ }^{\star} P<0.05 ;{ }^{* \star} P<0.01$. (G) Dko and WT KSL cells were transduced with a bicistronic retroviral vector encoding DN Stat5, DN Stat3, or empty vector, together with GFP, and cultured in medium containing SCF, FIt3 ligand, and IL-11. Ratios of cell numbers normalized against those of empty vector-transduced cells are plotted. For example, "WT (DNStat5/vec)" indicates the ratio of the numbers of WT cells transduced by DN Stat5 to those of WT cells transduced by empty vector. $\# P<0.05$ versus WT (DNStat5/vec). (H) Transduced KSL cells (150 cells seeded) were cultured in methylcellulose medium containing IL-3, IL-6, SCF, and erythropoietin. BFU, BFU-E; G, G-CFU; M, M-CFU; GM, GM-CFU; Mix, Mix-CFU.

and dko KSL cells, using a bicistronic retrovirus encoding DN Stat5 (DN Stat3 or empty vector as controls) and green fluorescent protein (GFP). As expected, DN Stat5 expression induced a decrease in mRNA expression of CIS, pim-1, and oncostatin $M$ (Figure 4F, lower panel). DN Stat5 suppressed in vitro proliferation of GFP-expressing dko KSL cells (designated as dko KSL/DNStat5 cells) more strongly than that of WT KSL cells (Figure 4G), demonstrating that dko KSL cells not only require Stat 5 activity for their growth, but they are also more sensitive to Stat 5 inhibition than WT cells. By contrast, DN Stat3 had little effect on the prolif- eration of dko or WT KSL cells. Colony-forming assays showed a drastic reduction of myeloid colony (M-CFU and G-CFU) formation by dko KSL/DNStat 5 cells, whereas dko KSL/DNStat 3 cells exhibited a capacity to form various myeloid colonies similar to dko KSL/vector cells (Figure 4H). Next, the transduced dko KSL cells were transferred to irradiated C57BL/6-Ly5.1 mice. We monitored donor-derived cells in peripheral blood at 3 and 9 weeks. As loss of Stat 5 was shown to result in reduced HSC engraftment (29), proportions of donor-derived cells in dko KSL/DNStat5 recipient mice were 3- to 4-fold lower than in dko KSL/vector recipient mice 


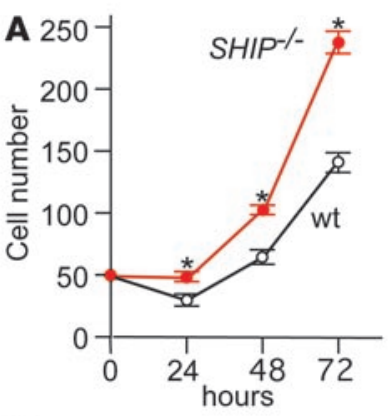

B

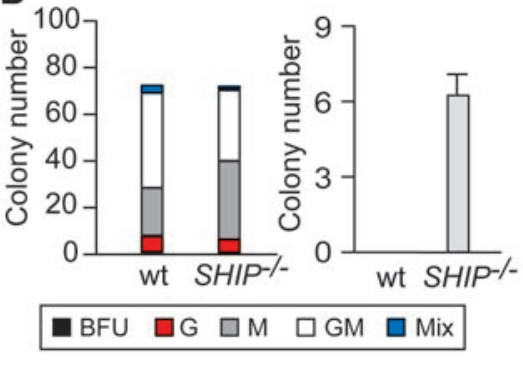

C
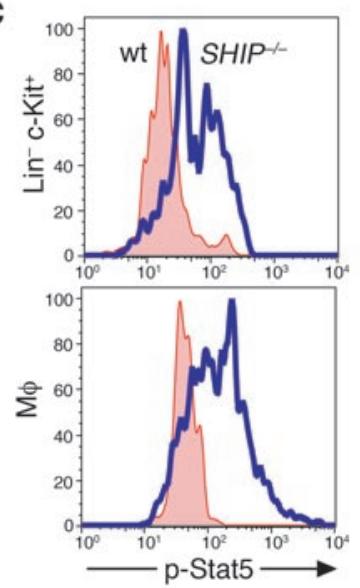

D
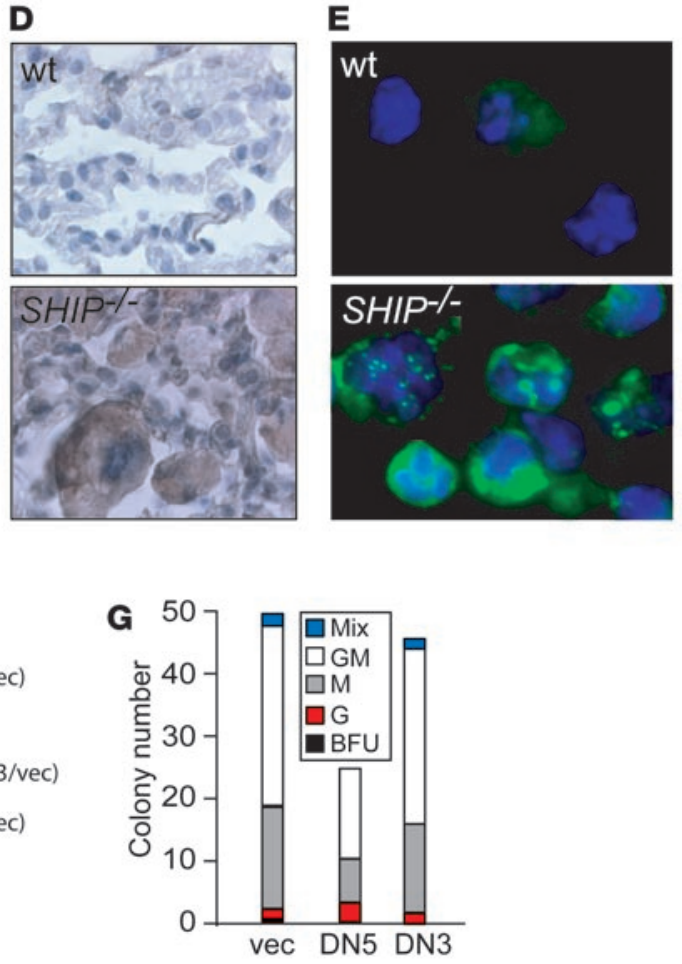

\section{Figure 5}

Stat5 activation is required for the increased proliferation and macrophage differentiation of $\mathrm{SHIP}^{-1-}$ KSL cells. (A) KSL CD34- cells derived from WT and $S H I P^{-/-}$mice were sorted into a 96-well plate containing SCF, Flt3 ligand, and IL-11 and cultured for 3 days. ${ }^{\star} P<0.05$ between WT and mutant. (B) Colony-forming assays showed skewed differentiation of KSL cells of SHIP ${ }^{-/-}$mice into macrophages in the presence of IL-3, IL-6, SCF, and erythropoietin (left panel). SHIP-/- KSL cells formed colonies in the absence of growth factors (right panel). (C-E) Stat5 in SHIP-IC-Kit+ Lin $^{-}$cells (C) and F4/80+ lung macrophages (C-E) was constitutively activated, as shown by flow cytometry (C), immunohistochemistry (D), and confocal microscopy (E) using anti-p-Stat5 (Tyr694). Original magnification, $\times 400$ (D and E). (F and G) SHIP ${ }^{-1-}$ and WT KSL cells were transduced with a bicistronic retroviral vector encoding DN Stat5, DN Stat3, or empty vector, together with GFP, and cultured in SCF, Flt3 ligand, and IL-11. Ratios of cell numbers normalized against those of empty vector-transduced cells are plotted. ${ }^{\#} P<0.05$ compared with cells transduced with empty vector. (F). Transduced cells (150 cells seeded) were cultured to form colonies in methylcellulose medium containing IL-3, IL-6, SCF, and erythropoietin (G).

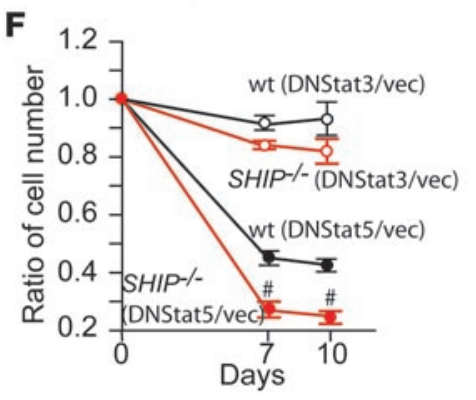

$(10.0 \% \pm 0.8 \%$ versus $33.0 \% \pm 13 \%$ at 3 weeks; $9.0 \% \pm 1.8 \%$ versus $38.0 \% \pm 14 \%$ at 9 weeks; $P<0.05$ at both time points). We also observed lower proliferation/differentiation of dko KSL/DNStat5 cells in the recipient mice, as $68.0 \% \pm 8.7 \%$ of the donor-derived cells were $\mathrm{CD}_{11} \mathrm{~b}^{+}$(granulocytes and/or monocytes) in dko KSL/ vector mice 9 weeks after transplantation, while only $21.4 \% \pm 6.2 \%$ $(P=0.002)$ were $\mathrm{CD} 11 \mathrm{~b}^{+}$in dko KSL/DNStat5 mice. Nine weeks after transplant, dko KSL/vector, but not dko KSL/DNStat5, mice developed MPD and lung inflammation (Table 1). Therefore, the lack of MPD in dko KSL/DNStat5 recipient mice can be due to the low engraftment or combined effects of low engraftment and low proliferation/differentiation of these cells. These results collectively demonstrate that Stat 5 activation is required for the increased proliferation and myeloid differentiation activity of dko HSCs, leading to the development of MPD and lung disease.

MPD and lung phenotypes and their dependence on Stat5 are shared by SHIP- and Lyn/Hck-deficient mice. Comparison of our analysis of Lyn/Hck-deficient mice with previous studies on $S H I P^{-1-}$ mice $(15,17,30)$, some of whose properties were confirmed in this study (Supplemental Figure 8), suggests that there is a phenocopy between lyn/hck dko and SHIP-/- mice in terms of MPD, lung disease, and perturbed hematopoiesis. HSCs and GMPs were increased, but common lymphoid progenitors were reduced in BM of SHIP $\mathrm{P}^{-/-}$mice (Supplemental Figure 9A). SHIP-/- HSCs also proliferated better than WT counterparts (Figure 5A), accompanied by accelerated cell cycling (Supplemental Figure 9B) in agreement with a recent study (12). SHIP-/- HSCs formed more monocyte/ macrophage colonies in the presence of cytokines than WT cells and also formed colonies spontaneously in the absence of growth factors (Figure 5B). The MPD and lung disease characterized by massive M2 macrophage infiltration were also transplantable with LT-HSCs from BM of SHIP-/- mice (Supplemental Figure 9D). Stat5 phosphorylation was increased in both HSCs and BAL macrophages from SHIP-/- mice (Figure 5, C-E). Consistent with this, mRNA expression of Stat 5 target genes was upregulated in SHIP-/- HSCs (Supplemental Figure 9C). Finally, introduction of DN Stat 5 into $S H I P^{-/-}$HSCs inhibited the proliferation and monocyte/macrophage colony formation of KSL cells (Figure 5, F and G). DN Stat 5 expression prevented the development of MPD and lung disease upon transplantation of $\mathrm{SHIP}^{-/-} \mathrm{KSL}$ cells (Supplemental Figure 9D), demonstrating that the MPD and lung disease in $S H I P^{-/-}$mice are also Stat5-dependent. 
A

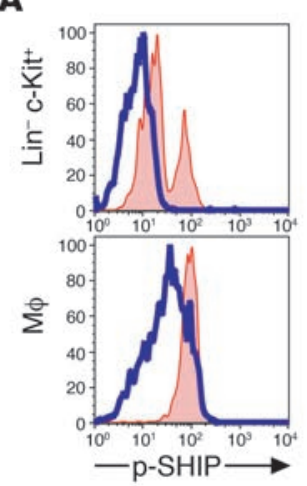

B

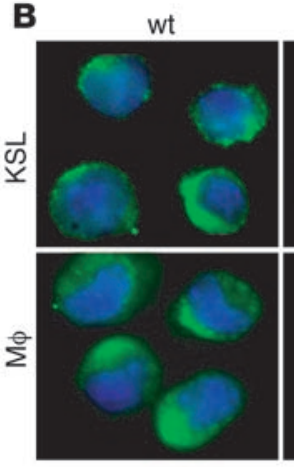

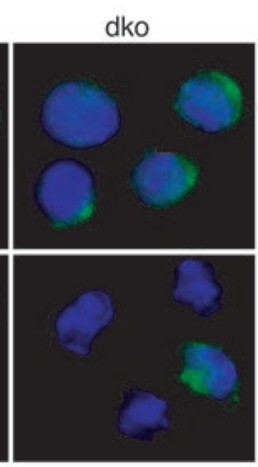

E

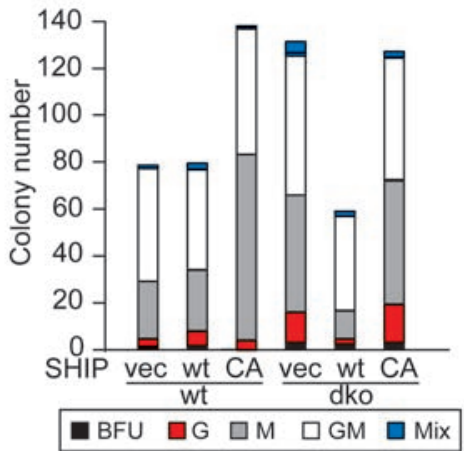

C
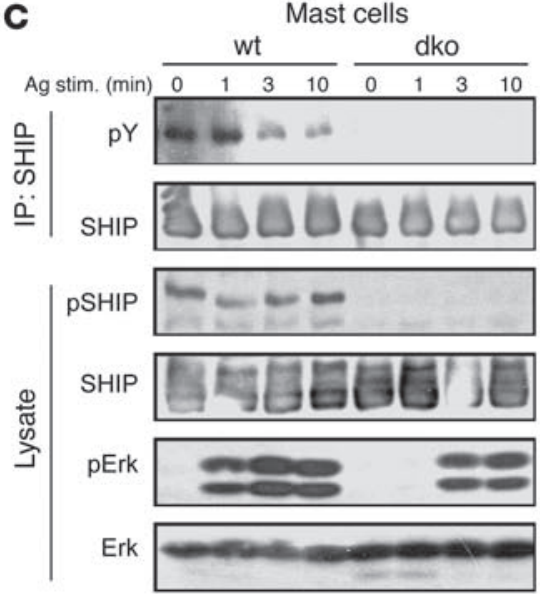

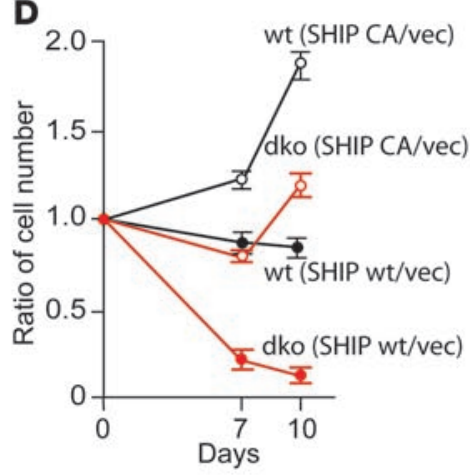

$\mathbf{F}$

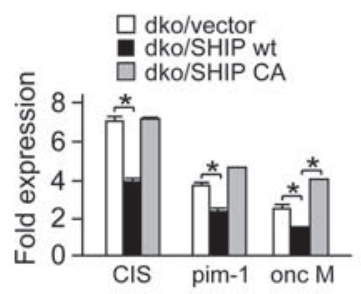

G

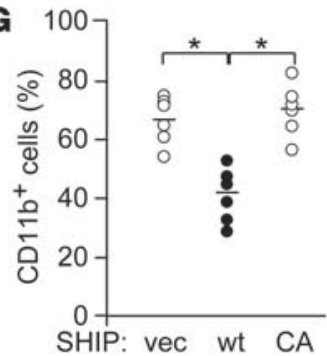

\section{Figure 6}

SHIP phosphorylation is reduced in Lyn/Hck-deficient HSCs and expression of a membrane-bound form of SHIP reverses dko phenotypes. (A and B) SHIP phosphorylation in KSL cells and macrophages was lower in dko than WT mice, as shown by flow cytometric analysis (A) and confocal microscopy (B) using anti-p-SHIP (Tyr1020). Original magnification, $\times 400$. (C) WT and dko BM-derived mast cells were sensitized with anti-DNP $\mathrm{IgE}$ and stimulated with antigen for the indicated periods. Tyrosine phosphorylation of SHIP was analyzed by immunoprecipitation followed by probing with anti-phosphotyrosine mAb (pY, upper panel) or cell lysates were directly analyzed by Western blotting using anti-p-SHIP (Tyr1020) antibody (lower panel). As a control to confirm activation of cells, blots were probed with anti-p-ERK1/2. (D and E) dko and WT KSL cells were transduced with a bicistronic retroviral vector encoding rCD2/SHIP WT, rCD2/SHIP C701A (CA), or empty vector, together with GFP, and cultured in medium containing SCF, Flt3 ligand, and IL-11. Ratios of cell numbers normalized against those of empty vector-transduced cells are plotted (D). Three days after retrovirus infection, some transduced cells (150 cells seeded) were cultured in methylcellulose containing IL-3, IL-6, SCF, and erythropoietin (E). (F) Real-time PCR analysis of mRNA expression of Stat5 target genes in dko KSL cells transduced with rCD2/SHIP WT or empty vector. (G) Dko KSL cells transduced with a bicistronic retroviral vector encoding rCD2/SHIP WT, rCD2/SHIP CA, or empty vector were i.v. injected into lethally irradiated mice. Eight weeks later, CD11b+GFP+ cells in peripheral blood were enumerated by flow cytometry. ${ }^{*} P<0.05$.

Expression of membrane-bound SHIP ameliorates or prevents MPD and lung disease in Lyn/Hck-deficient mice. The similarities between SHIP-1and Lyn/Hck-deficient mice raised the possibility that SHIP may be a mediator linking Lyn/Hck to activated Stat5. Indeed, we observed little SHIP phosphorylation in KSL, BAL macrophages, and BM-derived mast cells from dko mice (Figure 6, A-C), indicating that SHIP is a downstream target of Lyn and Hck. However, SHIP phosphorylation levels were comparable in $\mathrm{lyn}^{-/-}, \mathrm{bck}^{-/-}$, and WT mice (data not shown), suggesting that Lyn and Hck can compensate for each other and phosphorylate SHIP. The recruitment of SHIP to the plasma membrane is phosphorylation dependent and is a prerequisite for its function (31). Since SHIP phosphorylation and thus its membrane recruitment did not take place in dko cells, we reasoned that expression of a membrane-bound form of SHIP might reverse the phenotype of dko mice. To test this possibility, we took advantage of expression constructs encoding a rat CD2/SHIP fusion protein, in which the intracellular por- tion of rat CD2 was replaced by the SHIP phosphatase core of either WT or catalytically inactive (C701A) mutant enzyme. The fusion proteins were previously shown to constitutively reside in the plasma membrane (32). Expression of rCD2/SHIP WT in dko KSL cells inhibited their proliferative and colony (M-CFU and G-CFU) forming abilities (Figure 6, D and E). The development of lung disease was spared in recipient mice of $\mathrm{rCD} 2 / \mathrm{SHIP}$ WT-transduced dko KSL cells, although CD11 b cells in their peripheral blood were reduced but still higher than in WT mice (Figure 6G and data not shown). By contrast, rCD2/SHIP C701A did not inhibit the proliferative or colony-forming activities of dko HSCs or in vivo disease development. Interestingly, expression of $\mathrm{rCD} 2 / \mathrm{SHIP}$ C701A rather increased the proliferative and monocyte/macrophage colony-forming abilities of WT HSCs (Figure 6, D and E), indicating that $\mathrm{rCD} 2 /$ SHIP C701A has a DN function. These DN effects were not observed in dko cells, probably because SHIP functions in dko cells were already very low. 
A
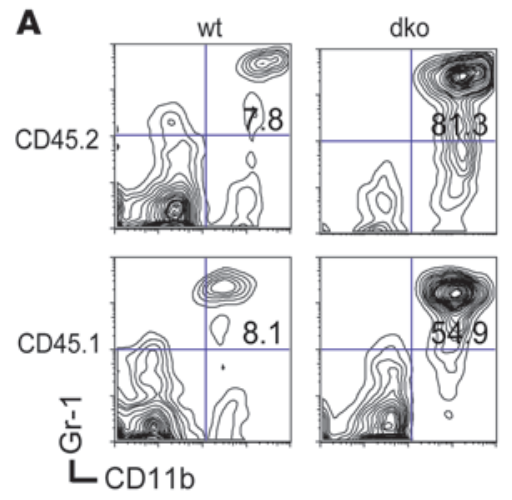

C
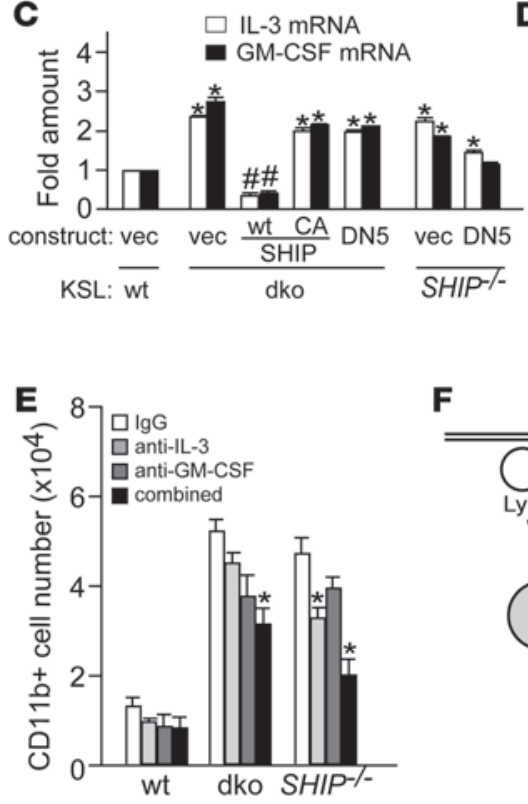

B
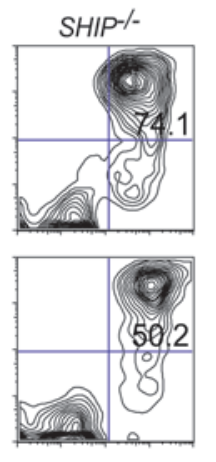

IL-3

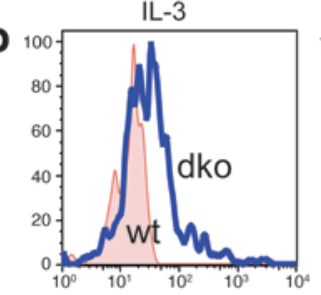

wt: $0.0 \pm 0.0 \%$ dko: $38.5 \pm 0.5 \%$
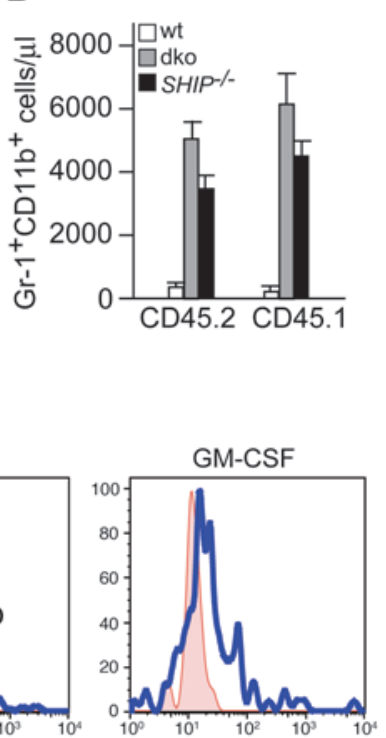

wt: $0.0 \pm 0.0 \%$

dko: $24.4 \pm 0.4 \%$

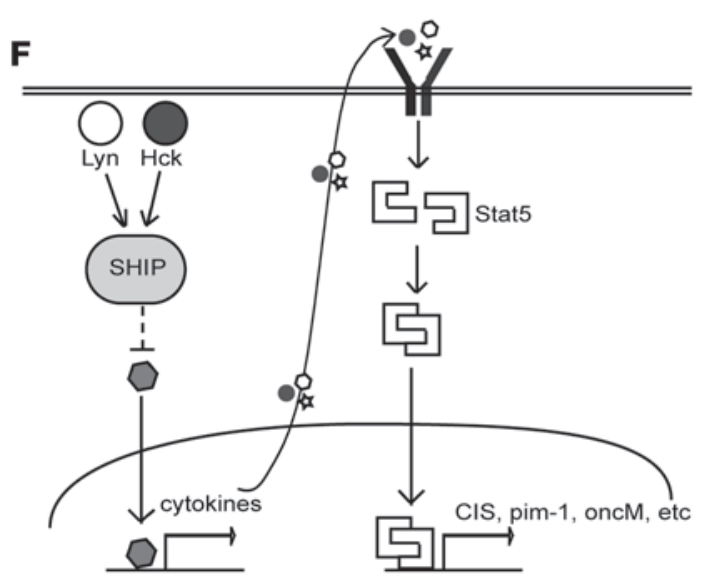

Figure 7

Cytokines secreted from dko and SHIP $P^{-/-}$cells promote in vitro and in vivo expansion of myeloid cells in a autocrine/paracrine manner. (A and $\mathbf{B}$ ) In vivo paracrine effect of dko and $S H I P^{-/-}$cells. Equal numbers of CD45.1 $1^{+}$ (Ly5.1) BM cells and CD45.2+ (Ly5.2) BM cells derived from either WT, dko, or SHIP ${ }^{-1-}$ mice were cotransferred into lethally irradiated Ly5.1 recipients. Eight weeks later, CD45.1 or CD45.2 cells in peripheral blood were gated and $\mathrm{Gr}-1{ }^{+} \mathrm{CD} 11 \mathrm{~b}+$ myeloid cells were enumerated. Percentages of $\mathrm{Gr}-1{ }^{+} \mathrm{CD} 11 \mathrm{~b}^{+}$ cells within each CD45 cohort are indicated. (C) RNAs from WT, dko, or SHIP-/- KSL cells transduced with the indicated bicistronic vectors were submitted to real-time PCR analysis. ${ }^{*} P<0.05$ versus WT/vector, ${ }^{\#} P<0.05$ versus $\mathrm{dko} /$ vector values, by Student's $t$ test. (D) Intracellular staining of IL-3 and GM-CSF in WT and dko KSL cells. (E) Lin- cells (Ly5.1) were cocultured for a week with either WT, dko, or SHIP-/- Lin- cells $\left(\right.$ Ly5.2+ ${ }^{+}$) in the presence of control IgG, anti-IL-3, anti-GM-CSF, or anti-IL-3 plus anti-GM-CSF. CD11 b+ cells were enumerated by flow cytometry. (F) A proposed model. Both Lyn and Hck are required to fully activate SHIP that in turn inhibits the production of several cytokines. In the absence of Lyn and Hck, secreted cytokines, including IL-3 and GM-CSF, can activate the Jak2/Stat5 pathway, eventually disrupting the homeostasis of HSCs.
Furthermore, rCD2/SHIP WT expression downregulated mRNA expression of Stat 5 target genes (Figure 6F), suggesting that the activity of Stat 5 in dko HSCs was at least partially inhibited by rCD2/SHIP WT. Therefore, we concluded that SHIP is a mediator linking Lyn/Hck to Stat5.

IL-3 and GM-CSF secreted from Lyn/Hck-and SHIP-deficient HSCs/ progenitors promote the expansion of myeloid cells. The above results suggest that Lyn and Hck activate SHIP and that SHIP in turn inhibits Stat5 activity. Previous studies showed that SHIP negatively regulates the production of several cytokines $(15,33)$. Therefore, it is possible that SHIP either directly inhibits Stat 5 activity or indirectly inhibits Stat 5 activity by inhibiting the secretion of a cytokine(s) that induces the activation of Stat 5 by an autocrine or paracrine mechanism. Direct SHIP-dependent regulation of Stat5 activity seems unlikely, as expression of rCD2/SHIP WT or C701A did not affect IL-3-induced Stat5 phosphorylation in Ba/F3 mouse pro-B cells (data not shown). Consistent with the paracrine mechanism, cotransfer of dko or SHIP-/- BM cells (Ly5.2) together with WT BM cells (Ly5.1) into irradiated Ly5.1 mice revealed that $\mathrm{dko}$ and $\mathrm{SHIP}^{-/-}$cells can promote the expansion of myeloid cells that differentiated from cotransferred Ly5.1+ WT cells (Figure 7, A and B). We also detected increased mRNA expression of several cytokines including IL-3, GM-CSF and G-CSF in dko KSL cells (data not shown). Importantly, transduction with rCD2/SHIP WT, but not rCD2/SHIP C701A, abrogated the expression of these cytokines in dko KSL cells (Figure 7C). By contrast, transduction with DN Stat 5 only slightly reduced the expression of these genes in dko and SHIP-/- KSL cells, indicating that the increased expression of these cytokine genes is largely independent of Stat 5 activity. Intracellular staining showed that KSL and lineage- $e^{-}$but not lineage ${ }^{+}$, cells of dko mice express IL-3 and GM-CSF (Figure 7D and data not shown). IL-3 and GM-CSF have been known to activate Stat5 (34). Incubation with IL-3 or GM-CSF promotes myeloid differentiation and proliferation of lineage ${ }^{-}$cells in our culture conditions (data not shown). To test whether IL-3 and GM-CSF secreted from dko or SHIP-/- cells are involved in the myeloid expansion of cotransferred WT Ly5.1 cells, we cocultured lineage- Ly5.1 cells with either WT, dko or SHIP-/cells (Ly5.2) in vitro. One week later, CD11b+ cells in the Ly5.1 pool were increased by approximately 3 -fold when cocultured with dko and $\mathrm{SHIP}^{-/-}$cells compared with those cocultured with Ly5.2+ WT cells (Figure 7E). Addition of anti-IL-3 or anti-GM- 
CSF neutralizing antibodies alone slightly reduced the numbers of CD $11 \mathrm{~b}^{+}$cells in the Ly5.1 pool, but the inhibitory effect of the combined antibodies was more substantial, reaching $40 \%-50 \%$ inhibition (Figure 7E). This inhibition could be larger, as control experiments showed that the antibodies we used blocked IL-3and GM-CSF-induced expansion of CD $11 \mathrm{~b}^{+}$cells at efficiencies of $76 \%$ and $72 \%$, respectively. These results demonstrated that paracrine secretion of IL-3 and GM-CSF is largely responsible for the expansion of myeloid cells of Ly5.1+ WT in cocultures with dko and SHIP ${ }^{-1-}$ cells. Furthermore, anti-IL-3 and anti-GM-CSF antibodies also inhibited growth factor-independent myeloid colony formation from dko BM cells (Figure 3F).

\section{Discussion}

In this study we revealed a novel signaling pathway that when perturbed can lead to myeloproliferative transformation: Lyn and Hck activate SHIP; active SHIP in turn inhibits autocrine or paracrine production of cytokines including IL-3 and GM-CSF, which, without upstream control, would lead to chronically activated Stat5 signaling (Figure 7F). This pathway is essential for the homeostasis of HSCs. We demonstrated that deficiency in Lyn/Hck or SHIP results in increased proliferation, growth factor-independent growth, and myeloid-skewed differentiation of HSCs, eventually generating transformed myeloid cells. Both the MPD and lung disease in Lyn/Hck- and SHIP-deficient mice seem to originate from the LT-HSC compartment.

SFKs can be activated by various cytokines and growth factors including IL-3 and GM-CSF. Previous studies support the sensitivity of this pathway to myeloproliferative transformation: GM-CSFtransgenic mice develop an extensive accumulation of macrophages in various organs (35). Transgenic mice expressing constitutively active mutants of the common $\beta$ subunit of the receptors for IL-3, IL-5, and GM-CSF induce MPD $(36,37)$. Interestingly, adoptive transfer of Nf1 tumor suppressor-deficient fetal liver cells induces MPD, a mouse model of human juvenile myelomonocytic leukemia, and GM-CSF and the common $\beta$ receptor subunit are involved in establishing and maintaining this $\operatorname{MPD}(38,39)$. Constitutively active Stat 5 in KSL CD34- cells can induce a fatal MPD (40). Several oncogenes, including BCR-ABL, FLT3 ITD, and JAK2 V617F, can induce MPD or frank leukemia in part by activating Stat5 at HSC levels (41-46). Interestingly, both the growth and monocyte/macrophage colony-forming activities in dko and SHIP ${ }^{-/-}$HSCs depend more heavily on Stat 5 than in WT HSCs. This phenomenon may represent a case of "oncogene addiction" of the mutant cells (47).

As described in the Introduction, Lyn and Hck play negative regulatory roles in myeloid cell differentiation in mice. Unlike lyn/hck dko mice, lyn/hck/fgr triple knockout mice did not develop MPD or dramatic abnormalities in hematopoiesis (48). This might suggest that Fgr antagonizes our proposed pathway (Figure 7), although it is not known at what level Fgr interferes with the pathway. However, it is possible that Fgr antagonizes Lyn and Hck by an unknown mechanism. As these SFKs, SHIP, cytokines, and Stat 5 are all conserved in humans, our proposed pathway is likely operative in human hematopoietic cells. However, gaps in our knowledge of SFKs' function in hematopoiesis are illustrated in their pathogenetic role in human chronic myelogenous leukemia (CML): both human LYN and HCK could phosphorylate BCR-ABL and contribute to CML (49-51). Furthermore, imatinibresistant $\mathrm{BCR}-\mathrm{ABL}^{+} \mathrm{K} 562$ cells expressed a higher level of LYN, and knocking down of LYN could reverse this resistance $(52,53)$. In a mouse model, however, Lyn, Hck, and Fgr are required for BCR-ABL-induced B cell acute lymphoblastic leukemia, but not BCR-ABL-induced CML (54). By contrast, our data demonstrated that Lyn and Hck are important negative regulators for a CML-like disease (e.g., MPD) in mice, raising such questions as whether in vivo functions of LYN and HCK in human CML are largely different from their in vitro functions or whether the roles of Lyn and Hck are species-specific. These issues are important to address in light of recent applications of SFK inhibitors in the treatment of imatinib-resistant or -intolerant $\operatorname{CML}(55,56)$.

Similar to SHIP, phosphorylation of SHP-1 is also Lyn-dependent $(57,58)$, and it was totally lost in dko cells (data not shown). SHP-1 can interact with, and inhibit phosphorylation of, Stat5 (59-61). Importantly, motheaten mice with homozygous mutations in the SHP-1 gene have a phenotype similar to dko and $S H I P^{-/-}$mice, in terms of MPD and lung pathology $(62,63)$. It will be interesting to investigate whether SHP-1 plays any role in the pathogenesis of the MPD and lung disease in dko mice.

Lung inflammation appears to be a cause of premature deaths in dko and $\mathrm{SHIP}^{-/-}$mice. The lung disease is characterized by a dramatic increase in M2 macrophages within alveoli. M2 macrophages are involved in debris scavenging, angiogenesis, tissue remodeling, wound healing, and the promotion of type II immunity, whereas classically activated (M1) macrophages eradicate invading microorganisms and tumor cells and promote type I immune responses $(13,14)$. Thus, M2 macrophages in dko and SHIP-/- lungs likely contribute to the pathogenesis of lung disease. However, little is known about the mechanisms underlying macrophage programming, and it is unresolved whether M1 and M2 macrophages represent distinct, defined lineages or whether mature macrophage phenotypes are plastic and adaptable to ever-changing environments. Our data confirmed and extended a previous study (15) by indicating that Lyn/Hck and SHIP are important for M2 macrophage programming. Increased Stat 5 activity may also play a role in promoting M2 macrophage differentiation, since constitutively active Stat 5 could induce $\mathrm{Ba} / \mathrm{F} 3$ cells to differentiate into macrophages (64), and Stat 5 was reported to be required for FDC-P1 cell differentiation into monocytes (65).

\section{Methods}

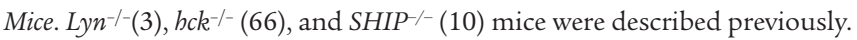
Dko mice were bred from $l y n^{-/-}$and $h c k^{-/-}$mice. These mice were backcrossed with C57BL/ 6 mice for 15 or more generations. The CD45 congenic mouse strains C57BL/6-Ly5.1 mice were purchased from The Jackson laboratory. Animal studies were approved by the institutional review board of La Jolla Institute for Allergy and Immunology.

Identification and purification of HSCs and progenitors. Cell sorting was performed by immunomagnetic-based pre-enrichment followed by multicolor flow cytometric sorting (see Supplemental Data for details).

In vitro cultures and colony-forming assays. LT-HSCs or $\mathrm{Lin}^{-}$cells were sorted into a 96 -well round bottom plate ( 1 cell per well) in $200 \mu \mathrm{l}$ of IMDM containing $5 \%$ FBS, $50 \mu \mathrm{M}$ 2-mercaptoethanol, and a cocktail of SCF, IL-11, and Flt 3 ligand. For myeloid colony-forming assays, BM cells or HSCs were cultured in Methocult M3434 (StemCell Technologies). For lymphoid colony-forming assays, BM cells were cultured in Methocult M3630 (StemCell Technologies). After 7-12 days, colonies were scored by microscopy, Wright-Giemsa staining, and flow cytometric analysis.

Transplantation of hematopoietic cells. BM cells were injected into lethally ( $9.6 \mathrm{~Gy})$ irradiated recipient mice. For transplantation of HSCs and pro- 
genitors, sorted LT-HSCs, ST-HSCs, CMPs, GMPs, and retrovirally transduced HSCs were injected into lethally irradiated mice together with Sca-1depleted C57BL/6-Ly5.1 helper BM cells.

Statistics. Two-tailed Student's $t$ test was used throughout this study. A $P$ value of less than 0.05 was considered significant.

\section{Acknowledgments}

We thank Gerald Krystal, Hiromitsu Nakauchi, and Stephen G. Ward for providing reagents. This study was supported in part by grants from the National Institutes of Health to T. Kawakami.
This is Publication 881 from the La Jolla Institute for Allergy and Immunology.

Received for publication September 22, 2007, and accepted in revised form November 28, 2007.

Address correspondence to: Toshiaki Kawakami, Division of Cell Biology, La Jolla Institute for Allergy and Immunology, 9420 Athena Circle, La Jolla, California 92037, USA. Phone: (858) 752-6814; Fax: (858) 752-6986; E-mail: toshi@liai.org.
1. Thomas, S.M., and Brugge, J.S. 1997. Cellular functions regulated by Src family kinases. Annu. Rev. Cell Dev. Biol. 13:513-609.

2. Nishizumi, H., et al. 1995. Impaired proliferation of peripheral $\mathrm{B}$ cells and indication of autoimmune disease in lyn-deficient mice. Immunity. 3:549-560.

3. Chan, V.W., Meng, F., Soriano, P., DeFranco, A.L., and Lowell, C.A. 1997. Characterization of the B lymphocyte populations in Lyn-deficient mice and the role of Lyn in signal initiation and down-regulation. Immunity. 7:69-81.

4. Harder, K.W., et al. 2001. Gain- and loss-of-function Lyn mutant mice define a critical inhibitory role for Lyn in the myeloid lineage. Immunity. 15:603-615.

5. Xu, Y., Harder, K.W., Huntington, N.D., Hibbs, M.L., and Tarlinton, D.M. 2005. Lyn tyrosine kinase: accentuating the positive and the negative. Immunity. 22:9-18

6. Mermel, C.H., et al. 2006. Src family kinases are important negative regulators of G-CSF-dependent granulopoiesis. Blood. 108:2562-2568.

7. Zhang, H., Meng, F., Chu, C.L., Takai, T., and Lowell, C.A. 2005. The Src family kinases Hck and Fgr negatively regulate neutrophil and dendritic cell chemokine signaling via PIR-B. Immunity. 22:235-246.

8. Lioubin, M.N., et al. 1996. p150Ship, a signal transduction molecule with inositol polyphosphate5-phosphatase activity. Genes Dev. 10:1084-1095.

9. Tu, Z., et al. 2001. Embryonic and hematopoietic stem cells express a novel SH2-containing inositol 5 '-phosphatase isoform that partners with the Grb2 adapter protein. Blood. 98:2028-2038.

10. Helgason, C.D., et al. 1998. Targeted disruption of SHIP leads to hemopoietic perturbations, lung pathology, and a shortened life span. Genes Dev. 12:1610-1620.

11. Liu, Q., et al. 1999. SHIP is a negative regulator of growth factor receptor-mediated $\mathrm{PKB} /$ Akt activation and myeloid cell survival. Genes Dev. 13:786-791.

12. Desponts, C., Hazen, A.L., Paraiso, K.H., and Kerr, W.G. 2006. SHIP deficiency enhances HSC proliferation and survival but compromises homing and repopulation. Blood. 107:4338-4345.

13. Gordon, S., and Taylor, P.R. 2005. Monocyte and macrophage heterogeneity. Nat. Rev. Immunol. 5:953-964.

14. Mantovani, A., Sica, A., and Locati, M. 2005. Macrophage polarization comes of age. Immunity. 23:344-346.

15. Rauh, M.J., et al. 2005. SHIP represses the generation of alternatively activated macrophages. Immunity. 23:361-374.

16. Kogan, S.C., et al. 2002. Bethesda proposals for classification of nonlymphoid hematopoietic neoplasms in mice. Blood. 100:238-245.

17. Harder, K.W., et al. 2004. Perturbed myelo/erythropoiesis in Lyn-deficient mice is similar to that in mice lacking the inhibitory phosphatases SHP-1 and SHIP-1. Blood. 104:3901-3910.

18. Osawa, M., Hanada, K., Hamada, H., and Nakauchi, H. 1996. Long-term lymphohematopoietic reconstitution by a single CD34-low/negative hematopoietic stem cell. Science. 273:242-245.
19. Passegue, E., Wagner, E.F., and Weissman, I.L. 2004. JunB deficiency leads to a myeloproliferative disorder arising from hematopoietic stem cells. Cell. 119:431-443.

20. Yilmaz, O.H., et al. 2006. Pten dependence distinguishes haematopoietic stem cells from leukaemiainitiating cells. Nature. 441:475-482.

21. Zhang, J., et al. 2006. PTEN maintains haematopoietic stem cells and acts in lineage choice and leukaemia prevention. Nature. 441:518-522.

22. Sawyers, C.L., McLaughlin, J., and Witte, O.N. 1995. Genetic requirement for Ras in the transformation of fibroblasts and hematopoietic cells by the BcrAbl oncogene. J. Exp. Med. 181:307-313.

23. Skorski, T., et al. 1997. Transformation of hematopoietic cells by BCR/ABL requires activation of a PI-3k/ Akt-dependent pathway. EMBOJ. 16:6151-6161.

24. Chan, T.O., Rittenhouse, S.E., and Tsichlis, P.N. 1999. AKT/PKB and other D3 phosphoinositide-regulated kinases: kinase activation by phosphoinositide-dependent phosphorylation. Annu. Rev. Biochem. 68:965-1014.

25. Matsumoto, A., et al. 1997. CIS, a cytokine inducible $\mathrm{SH} 2$ protein, is a target of the JAK-STAT5 pathway and modulates STAT5 activation. Blood. 89:3148-3154.

26. Nosaka, T., et al. 1999. STAT5 as a molecular regulator of proliferation, differentiation and apoptosis in hematopoietic cells. EMBO J. 18:4754-4765.

27. Lilly, M., and Kraft, A. 1997. Enforced expression of the $\mathrm{Mr}$ 33,000 Pim-1 kinase enhances factor-independent survival and inhibits apoptosis in murine myeloid cells. Cancer Res. 57:5348-5355.

28. Yoshimura, A., et al. 1996. Mouse oncostatin M: an immediate early gene induced by multiple cytokines through the JAK-STAT5 pathway. EMBO J. 15:1055-1063.

29. Bunting, K.D., et al. 2002. Reduced lymphomyeloid repopulating activity from adult bone marrow and fetal liver of mice lacking expression of STAT5. Blood. 99:479-487.

30. Nakamura, K., et al. 2004. Src homology 2-containing 5-inositol phosphatase (SHIP) suppresses an early stage of lymphoid cell development through elevated interleukin- 6 production by myeloid cells in bone marrow. J. Exp. Med. 199:243-254.

31. Phee, H., Jacob, A., and Coggeshall, K.M. 2000. Enzymatic activity of the Src homology 2 domaincontaining inositol phosphatase is regulated by a plasma membrane location. J. Biol. Chem. 275:19090-19097.

32. Freeburn, R.W., et al. 2002. Evidence that SHIP-1 contributes to phosphatidylinositol 3,4,5-trisphosphate metabolism in $\mathrm{T}$ lymphocytes and can regulate novel phosphoinositide 3-kinase effectors. J. Immunol. 169:5441-5450.

33. Kalesnikoff, J., et al. 2002. SHIP negatively regulates IgE + antigen-induced IL- 6 production in mast cells by inhibiting NF-kappa B activity. J. Immunol. 168:4737-4746.

34. Mui, A.L., Wakao, H., O’Farrell, A.M., Harada, N., and Miyajima, A. 1995. Interleukin-3, granulocytemacrophage colony stimulating factor and interleukin-5 transduce signals through two STAT5 homologs. EMBO J. 14:1166-1175

35. Lang, R.A., et al. 1987. Transgenic mice expressing a hemopoietic growth factor gene (GM-CSF) develop accumulations of macrophages, blindness, and a fatal syndrome of tissue damage. Cell. 51:675-686.

36. D’Andrea, R.J., et al. 1998. Dysregulated hematopoiesis and a progressive neurological disorder induced by expression of an activated form of the human common beta chain in transgenic mice. J. Clin. Invest. 102:1951-1960.

37. McCormack, M.P., and Gonda, T.J. 1999. Myeloproliferative disorder and leukaemia in mice induced by different classes of constitutive mutants of the human IL-3/IL-5/GM-CSF receptor common beta subunit. Oncogene. 18:7190-7199.

38. Birnbaum, R.A., et al. 2000. Nf1 and Gmcsf interact in myeloid leukemogenesis. Mol. Cell. 5:189-195.

39. Kim, A., et al. 2007. Beta common receptor inactivation attenuates myeloproliferative disease in Nf1 mutant mice. Blood. 109:1687-1691.

40. Kato, Y., et al. 2005. Selective activation of STAT5 unveils its role in stem cell self-renewal in normal and leukemic hematopoiesis. J. Exp. Med. 202:169-179.

41. Ye, D., Wolff, N., Li, L., Zhang, S., and Ilaria, R.L., Jr. 2006. STAT5 signaling is required for the efficient induction and maintenance of CML in mice. Blood. 107:4917-4925

42. Rocnik, J.L., et al. 2006. Roles of tyrosine 589 and 591 in STAT5 activation and transformation mediated by FLT3-ITD. Blood. 108:1339-1345.

43. Kralovics, R., et al. 2005. A gain-of-function mutation of JAK2 in myeloproliferative disorders. N. Engl.J. Med. 352:1779-1790.

44. James, C., et al. 2005. A unique clonal JAK2 mutation leading to constitutive signalling causes polycythaemia vera. Nature. 434:1144-1148.

45. Baxter, E.J., et al. 2005. Acquired mutation of the tyrosine kinase JAK2 in human myeloproliferative disorders. Lancet. 365:1054-1061.

46. Levine, R.L., et al. 2005. Activating mutation in the tyrosine kinase JAK2 in polycythemia vera, essential thrombocythemia, and myeloid metaplasia with myelofibrosis. Cancer Cell. 7:387-397.

47. Weinstein, I.B., and Joe, A.K. 2006. Mechanisms of disease: Oncogene addiction - a rationale for molecular targeting in cancer therapy. Nat. Clin. Pract. Oncol. 3:448-457.

48. Meng, F., and Lowell, C.A. 1997. Lipopolysaccharide (LPS)-induced macrophage activation and signal transduction in the absence of Src-family kinases Hck, Fgr, and Lyn. J. Exp. Med. 185:1661-1670.

49. Lionberger, J.M., Wilson, M.B., and Smithgall, T.E. 2000. Transformation of myeloid leukemia cells to cytokine independence by Bcr-Abl is suppressed by kinase-defective Hck. J. Biol. Chem. 275:18581-18585.

50. Meyn, M.A., 3rd, et al. 2006. Src family kinases phosphorylate the Bcr-Abl SH3-SH2 region and modulate Bcr-Abl transforming activity. J. Biol. Chem. 281:30907-30916.

51. Naito, H., et al. 2006. In vivo antiproliferative effect of NS-187, a dual Bcr-Abl/Lyn tyrosine kinase inhibitor, on leukemic cells harbouring $\mathrm{Abl}$ kinase domain mutations. Leuk. Res. 30:1443-1446. 
52. Donato, N.J., et al. 2003. BCR-ABL independence and LYN kinase overexpression in chronic myelogenous leukemia cells selected for resistance to STI571. Blood. 101:690-698.

53. Ptasznik, A., Nakata, Y., Kalota, A., Emerson, S.G., and Gewirtz, A.M. 2004. Short interfering RNA (siRNA) targeting the Lyn kinase induces apoptosis in primary, and drug-resistant, BCR-ABL1(+) leukemia cells. Nat. Med. 10:1187-1189.

54. Hu, Y., et al. 2004. Requirement of Src kinases Lyn, Hck and Fgr for BCR-ABL1-induced B-lymphoblastic leukemia but not chronic myeloid leukemia. Nat. Genet. 36:453-461.

55. Hochhaus, A., et al. 2007. Dasatinib induces notable hematologic and cytogenetic responses in chronic-phase chronic myeloid leukemia after failure of imatinib therapy. Blood. 109:2303-2309.

56. Quintas-Cardama, A., et al. 2007. Dasatinib (BMS354825 ) is active in Philadelphia chromosome-positive chronic myelogenous leukemia after imatinib and nilotinib (AMN107) therapy failure. Blood.
109:497-499.

57. Smith, K.G., Tarlinton, D.M., Doody, G.M., Hibbs, M.L., and Fearon, D.T. 1998. Inhibition of the B cell by CD22: a requirement for Lyn. J. Exp. Med. 187:807-811.

58. Cornall, R.J., et al. 1998. Polygenic autoimmune traits: Lyn, CD22, and SHP-1 are limiting elements of a biochemical pathway regulating BCR signaling and selection. Immunity. 8:497-508.

59. Ram, P.A., and Waxman, D.J. 1997. Interaction of growth hormone-activated STATs with SH2containing phosphotyrosine phosphatase SHP-1 and nuclear JAK2 tyrosine kinase. J. Biol. Chem. 272:17694-17702.

60. Paling, N.R, and Welham, M.J. 2002. Role of the protein tyrosine phosphatase SHP-1 (Src homology phosphatase-1) in the regulation of interleukin3 -induced survival, proliferation and signalling. Biochem. J. 368:885-894.

61. Minoo, P., Zadeh, M.M., Rottapel, R., Lebrun, J.J., and Ali, S. 2004. A novel SHP-1/Grb2-dependent mecha- nism of negative regulation of cytokine-receptor signaling: contribution of SHP-1 C-terminal tyrosines in cytokine signaling. Blood. 103:1398-1407.

62. Shultz, L.D., Bailey, C.L., and Coman, D.R. 1983. Hematopoietic stem cell function in motheaten mice. Exp. Hematol. 11:667-680.

63. Shultz, L.D., Coman, D.R., Bailey, C.L., Beamer, W.G., and Sidman, C.L. 1984. "Viable motheaten," a new allele at the motheaten locus. I. Pathology. Am. J. Pathol. 116:179-192.

64. Kawashima, T., et al. 2001. STAT5 induces macrophage differentiation of M1 leukemia cells through activation of IL- 6 production mediated by NF-kappaB p65. J. Immunol. 167:3652-3660.

65. Piazza, F., Valens, J., Lagasse, E., and Schindler, C. 2000. Myeloid differentiation of FdCP1 cells is dependent on Stat5 processing. Blood. 96:1358-1365.

66. Lowell, C.A., Soriano, P., and Varmus, H.E. 1994. Functional overlap in the src gene family: inactivation of hck and fgr impairs natural immunity. Genes Dev. 8:387-398. 